\section{Heimat ist auf keiner Landkarte zu finden.}

\section{Was von Ernst Bloch für den Umgang mit einem heimtücki- schen Begriff zu lernen ist ${ }^{1}$}

\section{Beat Dietschy}

\section{Was macht die Rede von «Heimat» heute wieder brisant?}

«Heimat» ist ein deutscher Begriff, der im 19. Jahrhundert Karriere gemacht hat. Er spielte in der Romantik eine Rolle, nicht zuletzt im Zusammenhang mit der Ausbildung eines deutschen Nationalbewusstseins. Er ist, wie das im Aufkommen von Heimatkunde und Heimatbewegungen zu sehen ist, verbunden mit einer Entdeckung und oft auch Glorifizierung der eigenen Geschichte. Ursprünglich kommt das Wort vom germanischen «Heim» her, das Haus, Grundeigentum oder Erbsitz bedeutet.

Im heutigen Sprachgebrauch wird «Heimat» oft mit dem Geburtsort, dem Ursprungsland oder dem Wohnsitz einer Person verbunden. Das zeigt eine Umfrage, die 1999 in Deutschland gemacht wurde: danach meint Heimat für 31 Prozent der Befragten den Wohnort, für 27 Prozent den Geburtsort, für 25 Prozent die Familie, für sechs Prozent die Freunde und für 11 Prozent das Land. ${ }^{2}$ Heimat, so kann man daraus schliessen, ist also für die meisten das, wo ich «zu Hause» bin oder wo ich mich «zu Hause» fühle.

1 Vortrag vor Studierenden der Niigata-University in Niigata, Japan (14.11.18).

2 Eduard Spranger, Der Bildungswert der Heimatkunde, zit. nach Gerd Koch, Heimat, in: Beat Dietschy / Doris Zeilinger / Rainer E. Zimmermann (Hg.): Bloch-Wörterbuch. Leitbegriffe der Philosophie Ernst Blochs, Berlin/Boston 2012, 169.
«Heimat» hat aber viele Bedeutungen, die mitschwingen, wenn man im Deutschen das Wort braucht. Es ist ein Wort voller Emotionen, und es ist nicht einfach, es wörtlich zu übersetzen. Als ich es in einem japanischdeutschen Wörterbuch nachgeschlagen habe, fand ich Dutzende japanischer Übersetzungsmöglichkeiten.

Ähnliches geschieht in anderen Sprachen. Max Frisch hat einmal gesagt: «MY COUNTRY erweitert und limitiert Heimat von vornherein auf ein Staatsgebiet, HOMELAND setzt Kolonien voraus, MOTHERLAND tönt zärtlicher als Vaterland, das mit Vorliebe etwas fordert und weniger beschützt als mit Leib und Leben geschützt werden will, LA PATRIE, das hisst sofort eine Flagge - und ich kann nicht sagen, daß mir beim Anblick eines Schweizerkreuzes sofort und unter allen Umständen heimatlich zumute wird... ${ }^{3}$

Es gibt gewiss Äquivalente, aber das Wort ist so stark mit der deutschen Sprache und Geschichte verbunden, dass das, was anklingt, wenn man es benutzt, in einem Land wie Japan sicher nur schwer verständlich zu machen ist. Auf jeden Fall möchte ich zu Beginn kurz erläutern, warum ich ausgerechnet «Heimat» als Thema meines Vortrags ausgewählt habe. Es ist nicht, um Ihnen Schwierigkeiten zu bereiten, sondern vielmehr, weil ich selber und viele Menschen in Deutschland, der Schweiz oder Österreich damit gegenwärtig grosse Schwierigkeiten haben.

Eigentlich ist Heimat ein Wort, bei dem viele an ihre Kindheit denken. «Da war ich zu Hause, da fühlte ich mich geborgen und beschützt», mögen wir dabei denken. Aber oft meinen wir damit etwas, was gar nicht so war. Gerne vergolden wir das Frühere und erfinden eine Zeit, in der alles gut und in Ordnung war. Heimat hat also etwas mit einer «Regression» zu tun: man wünscht sich zurück in einen kindlichen Zustand der Geborgenheit.

3 Max Frisch, Die Schweiz als Heimat? Rede zur Verleihung des Grossen Schillerpreises: Hans Mayer (Hg.), Max Frisch. Gesammelte Werke in zeitlicher Folge, Bd. 6, Frankfur am Main 1976, 510.
Jahrbuch Diakonie Schweiz 3 (2019) - ISSN 2504-3994

Dieser Text ist lizenziert unter einer Creative Commons Namensnennung 4.0 International Lizenz (CC BY 4.0): (https://creativecommons.org/licenses/by/4.0/).
Jahrbuch Diakonie Schweiz 3 (2019) http://dx.doi.org/10.22018/JDS.2019.5 
Wie kommt es dazu? In der Gegenwart fühlen sich viele nicht «zu Hause», sondern in der Fremde. Da ist kein fester Wohnort mehr, an dem man ein Leben lang bleiben kann. Auch die Arbeit oder der Beruf werden nicht mehr ein Leben lang ausgeübt. Die Beziehungen, die Ehen zerbrechen. Ein Lebensgefühl der Überforderung breitet sich mit der zunehmenden Globalisierung, Individualisierung und Beschleunigung aus. Der Soziologe Zygmunt Bauman hat das als Erfahrung des Fremdseins, die uns zugemutet wird, beschrieben. Darüber gleich mehr.

Ich selber habe, als ich etwa 18 Jahre alt war, mein eigenes Lebensgefühl in einem Wort ausgedrückt gefunden, das mir damals sehr wichtig war: «Unbehaustheit». Dabei hatte ich sehr wohl ein Zuhause, hatte Eltern, ich lebte nicht auf der Strasse. Doch die Wirklichkeit, die ich antraf, lehnte ich ab. Das teilte ich natürlich mit vielen anderen. Es war 1968. Viele Jugendliche dachten damals in Deutschland, Frankreich, Mexiko, den USA und auch in der wohlhabenden Schweiz so: wir lehnten uns auf gegen eine Welt, die «verwaltet» war, gegen eine ungerechte Welt, in der Menschen verhungern, gegen den Vietnamkrieg, gegen den Neo-Kolonialismus, wir waren gegen Staaten und Systeme, die wir für all das verantwortlich machten.

Kurzum: wir suchten eine andere Gesellschaft, eine friedlichere, gerechtere Welt, eine, die wir zusammen aufbauen. Wir wollten andere Lebensformen, anders wohnen (Wohngemeinschaften kamen auf, Kommunen), freiere Beziehungen als die von monogamen Paaren, man wünschte sogar den «Tod der Familie» (David Cooper) herbei. Und ja: wir lehnten uns auch auf gegen Universitäten, Lehrpläne, Professoren (es waren fast nur Männer), die das alles unterstützten, was wir ablehnten. Vor allem in Deutschland kam hinzu, dass Studenten ihre Elterngeneration ablehnten, weil sie verdrängt hat, was im Nationalsozialismus geschah. Sie warfen den Älteren vor, sich nicht wirklich mit dem Faschismus auseinandergesetzt zu haben. «Ihr habt Eure Mitschuld am Nationalsozialismus, am 2. Weltkrieg und am Holocaust verdrängt».

Das ist jetzt 50 Jahre her, ist Geschichte. Vieles ist seither anders geworden, und es schien so, als wäre diese Vergangenheit überwunden. Aber seit einigen Jahren kommt einiges davon wieder auf, das für mich sehr besorgniserregend ist. Stehen wir, fragen sich manche, vor neuem Nationalismus und möglicherweise wieder Krieg? Im Februar des letzten Jahres wurde ich in Deutschland zu einer Tagung über «Die neuen Wirren des Nationalismus» eingeladen. Ich sollte über «neuen Nationalismus» und «Rechtspopulismus» ${ }^{4}$ sprechen, denn ich hatte mich mit dem deutschen Faschismus beschäftigt und mit einem Philosophen, der sich damit bereits in den 20er Jahren auseinandergesetzt hat: Ernst Bloch.

Allerdings ist dieses Revival überwunden geglaubter Zustände keineswegs nur ein deutsches Thema. Die Tagung fand einen Monat nach dem Amtsantritt von Donald Trump statt. In Frankreich waren Präsidentschaftswahlen im Gang, und man befürchtete einen Wahlsieg von Marine Le Pen, die sich selber stolz als Populistin bezeichnet und den Franzosen, wie sie sagt, «ihr Vaterland zurückgeben» will. Ähnliche rechtspopulistische oder sogar rechtsextreme Bewegungen sind in vielen europäischen Ländern stark am Wachsen. In Holland, Schweden oder Dänemark zum Beispiel. In Österreich, Polen, Ungarn, der Türkei und seit kurzem auch in Italien sind sie sogar in der Regierung. In Deutschland hat es die «AfD» (Alternative für Deutschland) soeben geschafft, in allen Bundesländern im Parlament vertreten zu sein, sie erreichte je nach Bundesland bis zu 20 Prozent der Wählerstimmen.

Worte wie Heimat oder Nation spielen bei allen diesen Parteien und Bewegungen eine Schlüsselrolle. Sie alle versprechen wie Trump, das eigene Land wieder «gross» zu machen. Die eigenen Interessen sollen über alle anderen und über die gemeinsamen Interessen der Menschheit oder des Planeten gestellt werden. Das Klimaabkommen z.B. wird von Trump abgelehnt, weil er die eigenen auf fossilen Brennstoffen beruhenden In-

4 Vgl. Beat Dietschy, Was macht nationalistische Bewegungen attraktiv? Zur Gefahr einer Ethnisierung von Demokratie: Georg Wenz / Klaus Kufeld (Hg.), Die neuen Wirren des Nationalismus - Zwischen Agonie und Auftrieb, Landau 2017, 13-52. 
dustrien und seine Wählerbasis erhalten will. Und es fällt auf: die «Hei mat», welche in diesen Diskursen konstruiert wird, ist immer eine Heimat gegen andere, eine, welche andere ausschliesst.

Das «Eigene» soll gegen die «Fremden» verteidigt werden, d. h. gegen Immigranten, aber auch gegen bereits im Land wohnende «Fremde», deren Eltern zugewandert oder geflüchtet sind. Ich muss leider sagen, dass mein Land, die Schweiz, dabei sogar eine Vorreiterrolle in Europa gespielt hat. Bereits 1970 gab es eine rechtspopulistische Massenbewegung, welche mit einer Verfassungsinitiative die sogenannte «Überfremdung» des Landes durch italienische oder spanische Gastarbeiter bekämpfen wollte.

Besorgniserregend ist für mich vor allem der (politisch erfolgreich geschürte) Fremdenhass, der heute in vielen Ländern Europas grassiert. Es ist kein Zufall, dass gerade dies das Kernthema der neuen nationalistischen Bewegungen geworden ist. Zygmunt Bauman hat schon vor Jahren darauf hingewiesen, dass der Xenophobie Fremdheits- oder Entfremdungserfahrungen zugrunde liegen. ${ }^{5}$ Damit meint er: an Fremden erleben wir, dass wir selber fremd sind. Ihnen können wir dafür die Schuld geben. Denn sie tauchen in unserm Land auf, als Flüchtlinge, als Migranten und lassen uns eine Fremdheit erleben, die uns Angst macht, indem sie uns an unsere eigenen Ängste erinnert.

Fremde Menschen sind erlebbar. Aber das, was uns Angst macht, ist nicht so sichtbar wie ein Mensch mit anderer Hautfarbe oder eine Frau, die von Kopf bis Fuss in einem schwarzen Kleid daher kommt. Die rasant fortschreitende technische Modernisierung der digitalen Revolution hingegen, die unsere Lebensweise vollkommen umwälzt, betrifft uns viel massiver, aber gewissermassen hinterrücks. Viele Jobs verschwinden oder verändern sich grundlegend. Was wir einmal gelernt haben, ist schon bald überholt. Und die Gesellschaft droht immer mehr in unverbundene, abgeschottete Teilbereiche zu verfallen. Immer mehr Menschen haben

$-$

5 Zygmunt Bauman, Moderne und Ambivalenz. Das Ende der Eindeutigkeit, Hamburg 1992,83 gleichzeitig verschiedene Jobs. Das führt dazu, dass ein Individuum, wie Bauman sagt, in jedem Moment seines Lebens gleichzeitig «mehrere solcher divergenter Welten» bewohnt, sodass es «aus jeder «entwurzelt ist und in keiner (zu Hauses. Man kann sagen, dass es [das Individuum] der universale Fremde istı. 6

Die neoliberale Globalisierungspolitik der letzten dreissig Jahre hat diese Veränderungen der Lebenswelt ermöglicht, verstärkt und beschleunigt. Insgesamt führen die dadurch ausgelösten Veränderungen bei vielen Menschen zu Verunsicherung und Angst vor sozialem Abstieg. Sie fürchten, zum Spielball von Prozessen zu werden, die sie kaum durchschauen und über die sie keine Macht haben. Rechtspopulisten wie Donald Trump nutzen das aus. Sie versprechen dem Volk und den einfachen Leuten: «wir werden Euch die Macht zurückgeben». Sie verstärken die Ängste namentlich vor der Zuwanderung Fremder und bieten sich gleichzeitig als die Retter im bewusst geschürten Chaos an. Die (berechtigte) Verlustangst insbesondere von Angehörigen der Mittelschicht verstärken sie mit Hassparolen gegen Fremde und einer Verteidigung des «Eigenen». Sie propagieren eine «Herr im eigenen Haus»-Politik nach dem Muster: Deutschland den Deutschen oder Frankreich den Franzosen. Sie setzen ein angeblich kulturell homogenes eigenes «Volk» voraus, das die längst bestehende kulturelle Vielfalt unserer Gesellschaften leugnet. «Wir holen uns unser Land und unser Volk zurück», verspricht Gauland, der Chef der deutschen AfD. All dies erinnert uns an die Weimarer Zeit und den Aufstieg des Nationalsozialismus.

\section{Ein kurzer Blick zurück auf den Nationalsozialismus}

So verstandene «Heimat» ist für viele attraktiv. Sie verspricht ein Wurzelgefühl für Entwurzelte. In den zwanziger Jahren des letzten Jahrhunderts

6 Bauman, Moderne (Anm. 5), 124.
Jahrbuch Diakonie Schweiz 3 (2019) - ISSN 2504-3994

CC by 4.0
Jahrbuch Diakonie Schweiz 3 (2019) http://dx.doi.org/10.22018/JDS.2019.5 
war das eine Botschaft, die viele angesprochen hat, die im Krieg oder danach den Boden unter den Füssen verloren hatten. Ein Zitat aus einer Publikation von 1923 unter dem harmlosen Titel Der Bildungswert der Heimatkunde mag zeigen, wie sich eine neue Mythologie rund um Grund und Boden entwickeln konnte: «Heimat ist erlebbare und erlebte Totalverbundenheit mit dem Boden. Und noch mehr: Heimat ist geistiges Wurzelgefühl». ${ }^{7}$ Das schrieb der bekannte, humanistischen Bildungsidealen verpflichtete Philosoph und Pädagoge Eduard Spranger. Zehn Jahre später, im April 1933, vertritt er die Ansicht, «der Wille zur Volkwerdung [...], der aus den Kriegserlebnissen zur Kraft geworden ist und der den grossen positiven Kern der nationalsozialistischen Bewegung ausmacht», sei darin zu finden, dass der «Sinn für den Adel des Blutes und für Gemeinsamkeit des Blutes» betont und «bodenständige Heimattreue» gefordert werde. ${ }^{8}$

Sie werden wissen, dass «Blut und Boden» zentrale Elemente der nationalsozialistischen Propaganda waren. Ihre Weltanschauung konstruierte ein imaginäres «germanisches Volk», das sich durch seine Rasse (sein «Blut») von anderen, «minderwertigen Rassen» unterscheide. Eine Rassenvermischung lehnte sie kategorisch ab. Der Boden, das Territorium sollte allein der arischen Rasse gehören. Weil aber seit jeher auch «Nicht-Arier» auf dem sogenannt deutschen Boden lebten, schufen die Nazis den Volkohne-Raum-Mythos. Das hiess: das deutsche Volk hat nicht genug Raum, es muss expandieren können. Vertreibung und Vernichtung der NichtArier, insbesondere der Juden, und Eroberung von Territorien in Osteuropa im Krieg waren die Folge.

Eduard Spranger, Der Bildungswert der Heimatkunde, zit. nach Gerd Koch, Heimat, in: Beat Dietschy / Doris Zeilinger / Rainer E. Zimmermann (Hg.): Bloch-Wörterbuch. Leitbegriffe der Philosophie Ernst Blochs, Berlin/Boston 2012, 170.

8 Eduard Spranger, März 1993, in: Die Erziehung, Bd. 8, Nr. 7, 1932/1933, 401-408, 403
Die Rassentheorien der Nationalsozialisten und der aggressive Nationalismus - wie er in der Rede vom Dritten Reich oder vom tausendjährigen Reich zum Ausdruck kam, wurden von bürgerlichen wie von linken Antifaschisten als menschenverachtende Ideologie kritisiert. Dennoch konnte Hitler mit seinen Mythen Massen begeistern. Wie also kann man ihn erfolgreich bekämpfen? Das war die Frage, die Ernst Bloch sich stellte. Kritik und Aufklärung allein reichte offensichtlich nicht aus. Damit wurden nur Intellektuelle, nicht aber die Massen der «kleinen Leute» erreicht. Und so hat er versucht, den Nationalsozialisten den Gebrauch von Begriffen wie «Boden» und «Heimat», «Nation» oder sogar «Reich» ${ }^{9}$ streitig zu machen.

Bloch tat dies in zahlreichen Zeitungsartikeln und dann auch in dem Buch Erbschaft dieser Zeit, das allerdings erst Ende 1934 in Zürich erschien. Er nennt den Nationalsozialismus eine betrügerische Kopie von Sozialismus, welche einen scheinbaren «Antikapitalismus» zur Rettung des Kapitalismus einsetze. Ein Hauptadressat seiner Kritik war die Linke, namentlich die kommunistische, welche es unterlassen habe, die ambivalenten Gefühlslagen der Menschen zu bearbeiten, die unter der herrschenden «Rationalität» leiden, die ihr Leben zerstört. Sie haben genug davon, von Wirtschaftszahlen zu hören und zu erleben, wie sie sich wegen der Hyperinflation nicht mehr das Lebensnotwendige kaufen können.

Eine Episode, die Bloch mir einige Male erzählt hat, veranschaulicht das: sie handelt von einer Grossveranstaltung im Berliner Sportpalast, in der ein kommunistischer und ein Nazi-Redner aufeinandertrafen. Nachdem der Kommunist stundenlang vom Grundwiderspruch, von Kapital und Durchschnittsprofitrate in Marx ' Kapital gesprochen hatte, dankte ihm der Nazi und wandte sich ans Publikum mit den Worten: «Was haben Sie jetzt gehört: Zahlen, Zahlen und wieder Zahlen. Was tun Sie den ganzen

9 Vgl. Ernst Bloch, Erbschaft dieser Zeit, Frankfurt a.M. 1962, 18.
Jahrbuch Diakonie Schweiz 3 (2019) - ISSN 2504-3994

CC by 4.0
Jahrbuch Diakonie Schweiz 3 (2019) http://dx.doi.org/10.22018/JDS.2019.5 
Tag, wenn Sie im Büro als kleiner Buchhalter sitzen? Zahlen schreiben. Da zeigt sich, dass Kommunismus und Kapitalismus nur die Kehrseiten der gleichen Medaille sind». Er fuhr fort: «Ich aber spreche zu Euch in höherem Auftrag!» Nämlich dem Hitlers. ${ }^{10}$ Blochs provokatives Fazit dazu lautet: «die Nazis sprechen betrügend, aber zu Menschen, die Kommunisten völlig wahr, aber nur von Sachen». ${ }^{11}$ Sie sprechen die Menschen damit nicht an. Im Gegenteil. Denn «der kapitalistische Betrieb staut «Seele», und sie will abfließen, ja, gegen die Öde und Entmenschung explodieren» ${ }^{12}$.

Bloch analysiert aber nicht bloss die Erfolgsrezepte der Nazipropaganda. Er prüft vielmehr, was von den Hoffnungsbildern, die zum irrationalen «Flimmer- oder Rauschbetrug des Faschismus» ${ }^{13}$ beitragen, zu retten wäre: «Leben», «Seele», «Heimat» und «Erde», ja selbst «Volksgemeinschaft» und «Drittes Reich» gehören dazu.

Dass solche Symbolbegriffe nicht kampflos dem Nationalsozialismus zu überlassen sind, steht für Bloch ausser Zweifel. Denn sie enthalten uneingelöste Versprechen von einem besseren, schöneren Leben. ${ }^{14}$

Bloch kritisiert also den abstrakten Rationalismus der Linken. Und er entwirft eine Art «Rationalismus des Irrationalen». Er entwickelt nämlich eine Philosophie, in der Hoffnungen, Wünsche und Sehnsüchte eine zentrale Rolle spielen. Und ebenso eine Konzeption von «Heimat», welche ganz anders ist als das traditionelle Verständnis - und doch Elemente daraus aufnimmt und verwandelt.

10 Rainer Traub / Harald Wieser (Hg.), Gespräche mit Ernst Bloch, Frankfurt am Main 1977, 198.

11 Bloch, Erbschaft (Anm. 9), 153.

12 Bloch, Erbschaft (Anm. 9), 58.

13 Bloch, Erbschaft (Anm. 9), 16

14 Vgl. Bloch, Erbschaft (Anm. 9), 18.

\section{Ernst Blochs Verständnis von Heimat}

Möglich wurde ihm das nur, weil ihm selber die romantische Heimatsuche nichts Fremdes war. Sie hat als Reaktion auf Industrialisierung und Frühkapitalismus ja bereits im 19. Jahrhundert das einfache und naturnahe Leben auf dem Lande zu ihrem Sehnsuchtsort gemacht. Im Marxismus wurde dies meistens als eine Flucht aus der hässlichen Wirklichkeit gebrandmarkt. Diese Kritik hat Ernst Bloch nie geteilt. Er hat sich im Gegenteil für das interessiert, was in Märchen oder mythischen Vorstellungen an Rettungs- oder Befreiungsphantasien im kollektiven Bewusstsein weiterlebt. Das Lied der Seeräuber-Jenny in der Dreigroschenoper von Brecht und Weill gehörte für ihn zu solchen Befreiungsmythen, welche das reale Empfinden von Unterdrückten zum Ausdruck bringen.

In seinem Buch Spuren hat er solche Stoffe gesammelt. Dazu gehören auch Erinnerungen an eigenes Erleben wie jene seltene Augenblickserfahrung, die einen «Einklang von innen und aussen» zum Inhalt hat, draussen auf dem Land. Dieses ist jedoch nicht blosse Landschaftsumgebung, es ist gewissermassen behaust, und auch ein Freund spielt dabei eine wichtige Rolle:

«Ich speiste einst mit diesem Mann, die Schüsseln waren abgetragen, die Bauerntochter, die seine Geliebte war, ging in die Küche. Wir Freunde uns schweigend gegenüber und rauchten die Pfeife, die Tabakwolke roch, wie wenn man binter Waldarbeitern hergeht, so kräftig und gut nach Zimt; draußen das weite bayrische Land mit Kuppelwolken am unbewegten Himmel, eine Fliege summte in der Stube, die Bauerntochter klapperte mit dem keäftigen Geschirr. Ein böchst heiteres Kreisen ging füblbar zwischen Drinnen und Draußen, Schein und Tiefe, Kraft und Oberfläche. «Hören Sies, sagte da mein Freund, uwie gut das Haus in Gang ist. Und man hörte die Ruhe, das richtig Eingehängte, wie es läuft, die woblbekannte Kameradschaft mit den Dingen, die jeder Gesunde fühlt, die Lebensluft um sie her und die taohafte Welt. So nabe und fast aus dem gelebten Augenblick heraus, so selber darin zubause genossen wir das LLand und brauchten nicht einmal eine Strecke wegzugehen, um 
es in voller Figur zu sehen. Wir lagen zwar im Bann, aber der schien gut freilich gehörte das menschliche Haus dazu.»15

Es wäre aber verfehlt, das nun schon als Bild für Blochs Heimatverständnis zu nehmen. Es gibt nur einen Hinweis darauf. Leicht könnte man es als eine rückwärtsgewandte sogenannt «romantische» Verklärung vergangener Zeiten missverstehen.

Für Bloch aber ist Heimat nicht etwas, das schon da ist, noch gar das, was - vielleicht - einmal war. Heimat ist für ihn nicht mit Herkunft und Vergangenheit, sondern mit der Zukunft verbunden. In diesem Sinne könnte man sagen, dass Heimat noch auf keiner Landkarte zu finden ist Blochs Heimatbegriff ist also ziemlich anders als der gewohnte. Er spricht von Heimat als etwas, «worin noch niemand wan». So schreibt er in den berühmten Schlusssätzen von Das Prinzip Hoffnung:

"Die Wurzel der Geschichte aber ist der arbeitende, schaffende, die Gegebenheiten umbildende und überholende Mensch. Hat er sich erfasst und das Seine obne Entäußerung und Entfremdung in realer Demokratie begründet, so entsteht in der Welt etwas, das allen in die Kindheit scheint und worin noch niemand war: Heimat.» 16

Auch im Vorwort des dreibändigen Werks heisst es: «das Grundthema der Philosophie, die bleibt und ist, indem sie wird, ist die noch ungewordene, noch ungelungene Heimat» ${ }^{17}$. Wenn ebenfalls auf der letzten Seite und im letzten Satz, ja sogar, nach einem Doppelpunkt, als letztes Wort «Heimat»

15 Ernst Bloch, Spuren, Frankfurt a.M. 1969, 163.

16 Ernst Bloch, Das Prinzip Hoffnung, Frankfurt a.M. 1959, 1628

17 Bloch, Hoffnung (Anm. 16), 8 . auftaucht, so unterstreicht das, welche zentrale Bedeutung Bloch dieser Thematik gibt.

Wie kommt er dazu? Haben wir es mit einer romantischen Idee zu tun, im Sinne von Novalis, der auf die Frage: «Wo gehn wir denn hin?» die Antwort gab: «Immer nach Hause». ${ }^{18}$ Doch wenn damit eine Rückkehrsehnsucht verbunden wäre, so kehrt Bloch diese gerade um: seine Sehnsucht bezieht sich dezidiert auf noch nicht Vorhandenes, nicht auf eine gewesene oder noch bestehende Verwurzelung.

Nimmt er damit ein christliches, auch jüdisches Narrativ auf? Dafür spricht einiges. Heinrich Böll soll einmal gesagt haben, «ein religiöser Mensch sei daran zu erkennen, dass er sich in dieser Welt nie ganz daheim fühlt - er hat das Bewusstsein, dass alles Positive, was er mit dem Beheimatetsein verbindet, noch nicht gegeben ist, aber kommen wird.» ${ }^{19}$

Um ein blosses Abwarten des Kommenden handelt es sich dabei jedoch nicht. Denn lesen wir die Sätze des Prinzip Hoffnung genauer, dann sehen wir, dass Bloch nicht einfach von der Zukunft erwartet, was in der Gegenwart entsprechend einer linearen Zeitenfolge lediglich noch nich da ist. Drei Aspekte scheinen mir für Blochs Verständnis von Heimat im Prinzip Hoffnung wesentlich:

3.1 Heimat als Resultat gesellschaftlicher Veränderungspraxis

Zunächst fällt auf, dass hier von Schaffen und Arbeit die Rede ist: «Die Wurzel der Geschichte ist der arbeitende Mensch». Man könnte das als Hommage an die Arbeiterbewegung verstehen, ja gar an den Proletkult des Sowjetmarxismus. Doch stammt diese Formulierung aus der in den

18 Novalis, Heinrich von Ofterdingen. Ein Roman, Stuttgart 1987, 164.

19 Vgl. Francesca Vidal, Heimat und Migration. Oder: Der Versuch, Heimat als etwas zu sehen, worin noch niemand war, URL: https://www.selma.ws/content/heimat-migration (abgerufen am 28.02.2019). 
USA publizierten Broschüre Freiheit und Ordnung (1947). ${ }^{20}$ In der DDRAusgabe des Prinzip Hoffnung ergänzt er dann den Satz, nicht ohne Spitze gegen die Herrschenden: «Die Wurzel der Geschichte aber ist der arbeitende, schaffende, die Gegebenheiten umbildende und überholende Mensch.» ${ }^{21} \mathrm{Das}$ Transformieren und Überschreiten des Gegebenen wird also betont, nicht nur das Arbeiten.

Interessant ist auch, wie er den Ausdruck «Wurzel» verwendet. Er spricht gerade nicht von Heimat als Verwurzeltsein, das heisst im Sinne von Abstammung, sondern von radikal 22 sein: «wenn Gesellschaft und Dasein radikal werden, das heißt sich an der Wurzel fassen», sagt er im Satz davor. Damit spielt er auf den bekannten Marxsatz an: «Radikal sein ist die Sache an der Wurzel fassen. Die Wurzel für den Menschen ist aber der Mensch selbst» ${ }^{23}$

Wenn Bloch vom schaffenden, das Gegebene umbildenden Menschen spricht, dann meint er menschliche Produktivität, Arbeitsvermögen, kollektive geschichtsbildende Kreativität im gesellschaftlichen Produktionsprozess. Er zielt auf den Menschen, der sich selbst - statt eines göttlichen Schöpfers - hervorzubringen sucht und heraussetzt. Der Mensch ist für Bloch «ein heimatstiftendes Wesen, ein sich selbst stiftendes performatives Wesen» ${ }^{24}$.

20 Ernst Bloch, Freiheit und Ordnung. Abriss der Sozial-Utopien, New York 1947, 190.

21 Ernst Bloch, Hoffnung (Anm. 16), 489 (Kursives von bd: die Ergänzungen gegenüber Bloch, Freiheit und Ordnung).

22 Der Wortstamm von «radikal» ist lat. radix, Wurzel.

23 Karl Marx, Kritik der Hegelschen Rechtsphilosophie. MEW 1, 385.

24 Koch, Heimat (Anm. 7), 168, vgl. 178.

\subsection{Heimat als Antwort auf Entfremdung}

Renate Künast von den deutschen Grünen hat einmal in einer Rede gesagt: «Heimat ist dort, wo ich selber Ursache bin und nicht Wirkungen ferner, anonymer Gewalten ausgesetzt bin» ${ }^{25}$. Das verweist aber darauf, wogegen sich der philosophische Heimatbegriff wendet: wie schon im Grunde bei Novalis ist er ein Kampfbegriff gegen die Entfremdung.

Er bezieht sich - wie in der Romantik - auf Entfremdung von der natürlichen, aber auch von der gebauten technischen Umwelt, auf Entfremdung von den Mitmenschen, von der eigenen Tätigkeit und ihrem Produkt, und schliesslich auf Entfremdung von sich selbst. Das ist in der Tat ein Thema, das Marx umfassend erkundet hat. Er hat herausgearbeitet, dass unter den Bedingungen der kapitalistischen Warenproduktion den Menschen ihre eigenen gesellschaftlichen Beziehungen als Beziehungen von Sachen gegenübertreten. Diese «Verdinglichung» erleben wir auch in der Sprache: so wird sehr oft davon gesprochen, dass «die Finanzmärkte positiv oder negativ auf diese oder jene Entscheidung reagieren» - als ob sie Personen wären. Was geschichtlich geworden, von Menschen gemach wurde, tritt ihnen als fremde, unbeherrschbare Macht entgegen.

Der Soziologe Hartmut Rosa hat dies in seinem jüngsten Buch als das Verlorengehen der «Resonanz» zwischen Ich und Welt beschrieben. ${ }^{26}$ Seine These ist: «Der vormoderne Mensch, soweit er in einer vorgegebenen, als Teil einer kosmologischen Ordnung erfahrenen Gesellschaftsform lebte, besetzte gleichsam einen [...] mit der Geburt definierten festen Platz in einer 〈großen Ordnung des Daseins〉. Mit der Moderne löst sich diese unverrückbare Bindung des Subjekts an Raum, Dinge und Menschen: Finde Deinen eigenen Platz in der Welt! wird zur Grundaufgabe des

25 Nach Koch, Heimat (Anm. 7), 171.

26 Hartmut Rosa, Resonanz. Eine Soziologie der Weltbeziehung, Berlin 2016.
Jahrbuch Diakonie Schweiz 3 (2019) - ISSN 2504-3994

CC by 4.0
Jahrbuch Diakonie Schweiz 3 (2019) http://dx.doi.org/10.22018/JDS.2019.5 
modernen (bürgerlichen) Subjekts.» ${ }^{27}$ Die Spätmoderne aber beschleunigt dies, wie schon erwähnt, enorm. Wohnorte, Berufsstellen, Lebensabschnittspartner, religiöse und politische Überzeugungen: nichts mehr ist lebenslang. Das hohe Tempo des sozialen Lebens der Spätmoderne vermehrt Rosa zufolge die Entfremdung. Es führe zum Fremdwerden des Raumes, der Dinge und Orte, der Menschen und Verhältnisse, «weil nichts mehr zur Heimat in dem Sinne gerinnt, dass es identitätsstiftende Bedeutung erlangts ${ }^{28}$.

Vor diesem Hintergrund von Verdinglichung und Entfremdung wird verständlicher, weshalb Bloch so sehr auf der Ortlosigkeit (Utopie) von Heimat insistiert: sie durch Identifikation mit etwas, was da ist, suchen zu wollen, verortet, territorialisiert sie. So «landet» man dann leicht in einer Apotheose des Gegebenen, sei es im real geglaubten Sozialismus oder in der Identifikation mit dem je eigenen Vaterland, Volk oder Führer.

Darum heisst es im Prinそip Hoffnung unmittelbar vor unseren Sätzen:

«Der Mensch lebt noch überall in der Vorgeschicbte, ja alles und jedes steht noch vor Erschaffung der Welt, als einer recbten. Die wirkliche Genesis ist nicht am Anfang, sondern am Ende, und sie beginnt erst anzufangen, wenn Gesellschaft und Dasein radikal werden, das heisst sich an der Wurzel fassen.» 29

Das bedeutet aber alles andere als ein Abwarten von Zukunft. Das «Utopische» wird bei Bloch konkret erst in humaner Praxis - in real werdender Demokratie: «Hat er [der Mensch] sich erfasst und das Seine ohne

27 Hartmut Rosa, Heimat im Zeitalter der Globalisierung, in: der blaue reiter. Journal für Philosophie, Heft 23, Hannover 2007, 15.

28 Rosa, Heimat (Anm. 27), 18

29 Bloch, Hoffnung (Anm. 16), 1628. Auch damit rekurriert Bloch auf Marx (vgl. MEW $13,9)$.
Entäusserung und Entfremdung in realer Demokratie begründet, so entsteht in der Welt [...] Heimat».

Daraus kann man folgern: Wenn Demokratie das Gestalten von Verhältnissen zwischen Menschen und von Menschen zur Natur betrifft, so lässt sich das Entstehen von Heimat daran ablesen, ob und wie das gelingt. Heimat ist dann kein Ort und kein Land auf einer Landkarte, sondern gelebte Demokratie. Sie ist nicht einfach gegeben, aber herstellbar, sie ist das Ergebnis von Prozessen, in denen sich Demokratie realisiert. Sie liegt in der Perspektive einer neuen gesellschaftlichen Organisationsform von Arbeit und gesellschaftlichen Beziehungen, die als friedliches Miteinander umschrieben werden können. Heimat meint Frieden, der wesentlich mehr als die Absenz von Krieg bedeutet.

Bloch liefert bewusst kein Heimatbild, an das wir uns halten könnten. Er pinselt keine Utopie aus. Doch das, was Heimat sein könnte, wird sichtbar in jedem Kampf um reale Demokratie, auch dem gescheiterten. Angesichts des Hitler-Regimes, das im Dritten Reich die verwirklichte Volksgemeinschaft sah, hat Bloch in Erbschaft dieser Zeit darauf insistiert: eine Volksgemeinschaft oder ein «Vaterland wird erst geboren durch Entfernung seiner Nutzniesser, durch reale Aufhebung der Klassen» ${ }^{30}$.

\subsection{Heimat als Vorgriff auf andere Naturverbältnisse}

Wir können also sagen: die «Vorgeschichte» endet erst, wenn Herrschaftsund Ausbeutungsverhältnisse verschwinden, und zwar auch jene gegenüber der Erde. Ernst Bloch ist einer der wenigen Philosophen, die im 20. Jahrhundert eine Naturphilosophie entwickelt haben. Er hat darauf insistiert, dass nur im Bund mit der Natur, nicht gegen sie das Experiment Menschenwelt eine Zukunft hat. Er hat darum auch für eine andere Technik, eine «Allianz-Technik» plädiert. Angesichts der Folgen der menschen-

30 Bloch, Erbschaft (Anm. 9), 99
Jahrbuch Diakonie Schweiz 3 (2019) - ISSN 2504-3994

CC by 4.0
Jahrbuch Diakonie Schweiz 3 (2019) http://dx.doi.org/10.22018/JDS.2019.5 
gemachten Klimaerwärmung und der Übernutzung aller natürlichen Ressourcen scheint mir gerade diese Seite seines Denkens sehr aktuell, auch wenn wir heute noch viel weiter gehen müssen mit dem Umdenken und der Neuorientierung unseres Wirtschaftens, als Bloch es voraussehen konnte.

Immerhin hat er schon deutlich hingewiesen auf das «Zukunftsproblem im tragenden, umfassenden Raum der Heimat: der Natuns ${ }^{31}$. Wir können wohl kaum bestreiten, dass wir das bei der Umgestaltung der gesellschaftlichen wie auch der Naturverhältnisse zu berücksichtigen haben. Denn «menschliche Freiheit und Natur als ihre konkrete Umgebung (Heimat) bedingen sich wechselseitig» ${ }^{32}$. Das Wirklichwerden von Heimat verspricht erst der Tag, an dem auch der kurzsichtig eigennützige Anthropozentrismus oder Human-Nationalismus («Humans first») gegenüber der Natur verschwunden sein wird und eine andere, «kosmo»-politische, das heisst, menschen- und naturgerechte Einstellung seinen Platz eingenommen hat.

Autor:

Beat Dietschy, Dr. phil., Theologe und Philosoph, Präsident COMUNDO, ehem.

Zentralsekretär Brot für alle

31 Bloch, Hoffnung (Anm. 16), 16. Darum beruft er sich auf der Schlussseite des Prinzips Hoffnung noch einmal auf Marx, indem er gegenüber der früheren Fassung diese Schlussätze (vgl. Anm. 20) den Satz einfügt: «Marx bezeichnet als sein letztes Anliegen «die Entwicklung der menschlichen Natur; dieser menschliche Reichtum wie der von Natur insgesamt liegt einzig in der Tendenz-Latenz, worin die Welt sich befindet - visà-vis de tout» (1628).

32 Bloch, Hoffnung (Anm. 16), 1080. 